\title{
Structure of $\left[\mathrm{Cu}(\mathrm{tssb})(\right.$ phen $\left.) \mathrm{H}_{2} \mathrm{O}\right] \cdot \mathrm{C}_{2} \mathrm{H}_{5} \mathrm{OH} \cdot 0.5 \mathrm{H}_{2} \mathrm{O}\left(\mathrm{H}_{2}\right.$ tssb $=$ Schiff Base Derived from Salicylaldehyde and Taurine, phen = 1,10-Phenanthroline)
}

\author{
Zheng LiU, ${ }^{* * *}$ Shu-Hua Zhang, ${ }^{* *}$ and Yuan-Bin LiN*† \\ *College of Chemistry Xiangtan University, Xiangtan, Hunan 411105, P. R. China \\ **Department of Materials and Chemistry Engineering, Guilin University of Technology, \\ Guilin, Guangxi, 541004, P. R. China
}

\begin{abstract}
A new complex, $\left[\mathrm{Cu}(\mathrm{tssb})(\right.$ phen $\left.) \mathrm{H}_{2} \mathrm{O}\right] \cdot \mathrm{C}_{2} \mathrm{H}_{5} \mathrm{OH} \cdot 0.5 \mathrm{H}_{2} \mathrm{O}$, crystallizes in the triclinic, space group $P \overline{1}$ with cell parameters $a$ $=10.593(2), b=11.600(2), c=21.170(4) \AA, \alpha=103.340(4), \beta=96.937(4), \gamma=105.056(3)^{\circ}, Z=4, V=2398.9(7) \AA^{3}$. Its crystal structure was determined and refined to a final $R=0.0468$ for 10894 independent reflections. The $\mathrm{Cu}$ (II) ion forms a distorted octahedral geometry by one nitrogen atom and two oxygen atoms from tssb, two nitrogen atoms from phen and one oxygen atom from water.
\end{abstract}

(Received April 19, 2006; Accepted August 14, 2006; Published on web October 24, 2006)

Schiff-base complexes containing sulfur and complexes of amino acid Schiff-bases ${ }^{1}$ have recently aroused increasing interest because of their antiviral, anticancer and antibacterial activities. Taurine, an amino acid containing sulfur, is indispensable to human beings, and has important physiological functions. Recently, we have found that the Schiff-base derived from taurine has manifold coordination modes. ${ }^{2}$ Aromatic-ring stacking interaction is an important characteristic of ternary complex, and has important functions in organism, such as stabilizing the double- helical structure ${ }^{3}$ and the interactions between anticancer drugs and DNA. ${ }^{4}$ Herein we chose $\mathrm{H}_{2} \mathrm{tssb}$, phen and $\mathrm{Cu}(\mathrm{Ac})_{2} \cdot 4 \mathrm{H}_{2} \mathrm{O}$ to synthesize a new compound, $\left[\mathrm{Cu}(\mathrm{tssb})\left(\right.\right.$ phen) $\left.\mathrm{H}_{2} \mathrm{O}\right] \cdot \mathrm{C}_{2} \mathrm{H}_{5} \mathrm{OH} \cdot 0.5 \mathrm{H}_{2} \mathrm{O}$ (see Fig. 1). The crystal structure of the title compound was determined using BRUKER SMART 1000 CCD. The experimental conditions, crystal data and structure refinement results are summarized in Table1; atomic coordinates and equivalent isotropic displacement parameters are listed in Table 2.

As shown in Fig. 2(a), the crystal contains two crystallographycally independent discrete molecules. The $\mathrm{Cu}$ (II) ion forms a distorted octahedral geometry by one nitrogen atom and two oxygen atoms from tssb ${ }^{2-}$, two nitrogen atoms from phen and one oxygen atom from terminal water, with $\mathrm{O}(1)$ and $\mathrm{O}(2)$ at the apical positions. The inter-atomic distances of $\mathrm{Cu}(1)-\mathrm{O}(1), \mathrm{Cu}(1)-\mathrm{N}(1), \mathrm{Cu}(1)-\mathrm{N}(2) \mathrm{Cu}(1)-\mathrm{O}(2)$, $\mathrm{Cu}(1)-\mathrm{N}(3)$ and $\mathrm{Cu}(1)-\mathrm{O}(1 \mathrm{~W})$ are 2.006(2), 2.101(3), 2.101(2), $2.139(2), 2.042(3)$ and 2.072(2) , respectively. Similar values have been observed in $\mathrm{Cu}$ (II) complex of salicylaldehyde Schiff-base. $^{2}$ As expected, all other bond distances and bond angles are within the normal ranges, in good agreement with those of other taurine-containing complexes. ${ }^{1-3}$ The coordination of $\mathrm{Cu}(2)$ is similar to that of $\mathrm{Cu}(1)$, but the corresponding bond lengths are slightly different $(\mathrm{Cu}(2)-\mathrm{O}(5)$ 2.2004(2) $\mathrm{A}, \mathrm{Cu}(2)-\mathrm{N}(4)$ 2.093(3) $\mathrm{A}, \mathrm{Cu}(2)-\mathrm{O}(2 \mathrm{~W}) 2.121(2) \AA$, $\mathrm{Cu}(2)-\mathrm{N}(5) 2.093(3) \AA, \mathrm{Cu}(2)-\mathrm{N}(6) 2.049(2) \AA$ and $\mathrm{Cu}(2)-\mathrm{O}(6)$ $2.097(2) \AA)$. The complex is linked by hydrogen bonds and

$\uparrow$ To whom correspondence should be addressed E-mail: Lisa4.6@163.com aromatic-ring stacking forming a three-dimensional structure (see Table 3 and Fig. 2(b)).

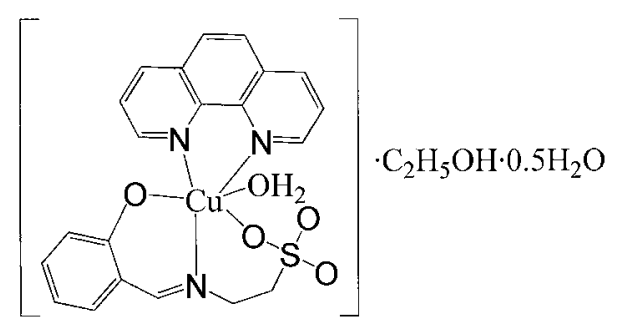

Fig. 1 Chemical diagram of the title compound.

Table 1 Crystal and experimental data

$\begin{array}{ll}\text { CCDC } & 612502 \\ \text { Formula } & \mathrm{C}_{23} \mathrm{H}_{26} \mathrm{CuN}_{3} \mathrm{O}_{6.50} \mathrm{~S} \\ \text { Formula weight } & 544.07 \\ \text { Crystal system } & \text { triclinic } \\ \text { Space group } & P \overline{1}, Z=4 \\ a=10.593(2) \AA & \alpha=103.340(4)^{\circ} \\ b=11.600(2) \AA & \beta=96.937(4)^{\circ} \\ c=21.170(4) \AA & \gamma=105.056(3)^{\circ} \\ V=2398.9(7) \AA^{3} & D_{\mathrm{c}}=1.506 \mathrm{~cm}^{-3} \\ \text { Linear absorption factor } & 1.044 \mathrm{~mm}^{-1} \\ F(000)=1128 & (\Delta / \sigma)_{\text {max }}=0.01 \\ \theta \text { range for data collection } & 1.01 \text { to } 27.5^{\circ} \\ \text { No. of reflections used with } & 7921 \\ I>2 \sigma(I) & \\ \text { No. of measured reflections } & 16295\left(R_{\text {int }}=0.0186\right) \\ R_{1}[I>2 \sigma(I)]=0.0468 & w R_{2}=0.1450 \\ \Delta \rho_{\text {max }}=0.940 \text { e } \AA^{-3} & \Delta \rho_{\text {min }}=-0.497 \text { e } \AA^{-3} \\ \text { Crystal dimensions [mm] } & 0.38 \times 0.36 \times 0.32 \\ \text { Measurement } & \mathrm{BRUKER} \mathrm{SMART} \mathrm{1000} \mathrm{CCD} \\ \text { Mo } K_{\alpha} & \lambda=0.71073 \AA \\ \text { Structure determination } & \text { direct methods }(\mathrm{SHELXS}-97)^{5} \\ \text { Refinement } & \text { full matrix least-squares } \\ & \left(\mathrm{SHELXL-97)^{5 }}\right. \\ & \end{array}$


Table 2 Atomic coordinates $\left(\times 10^{4}\right)$ and equivalent isotropic displacement parameters $\left[\AA^{2} \times 10^{3}\right]$ for the title compound

\begin{tabular}{|c|c|c|c|c|}
\hline Atom & $x$ & $y$ & $z$ & $U(\mathrm{eq})^{\mathrm{a}}$ \\
\hline $\mathrm{Cu}(1)$ & $815(1)$ & $1477(1)$ & $3304(1)$ & $30(1)$ \\
\hline$S(1)$ & $-915(1)$ & $3113(1)$ & $2706(1)$ & $36(1)$ \\
\hline$O(1 W)$ & $138(2)$ & $71(2)$ & $2428(1)$ & $40(1)$ \\
\hline$O(1)$ & $2219(2)$ & $702(2)$ & $3554(1)$ & $35(1)$ \\
\hline$O(2)$ & $.802(2)$ & $2172(2)$ & $3049(1)$ & $40(1)$ \\
\hline$O(3)$ & $-1615(3)$ & $3927(3)$ & $3030(1)$ & $57(1)$ \\
\hline$O(4)$ & $-1463(3)$ & $2564(3)$ & $2004(1)$ & $59(1)$ \\
\hline$N(1)$ & $1161(3)$ & $2758(3)$ & $4235(1)$ & $35(1)$ \\
\hline $\mathrm{N}(2)$ & $-466(2)$ & $437(2)$ & $3790(1)$ & $34(1)$ \\
\hline $\mathrm{N}(3)$ & $2101(2)$ & $2651(2)$ & $2923(1)$ & $31(1)$ \\
\hline C(1) & $1978(4)$ & $3888(4)$ & $4449(2)$ & $53(1)$ \\
\hline$C(2)$ & $2041(5)$ & $4705(4)$ & $5068(2)$ & $70(1)$ \\
\hline$C(3)$ & $1199(5)$ & $4311(4)$ & $5461(2)$ & $64(1)$ \\
\hline C(4) & $342(4)$ & $3087(4)$ & $5266(2)$ & $50(1)$ \\
\hline$C(5)$ & $-494(4)$ & $2553(5)$ & $5667(2)$ & $63(1)$ \\
\hline $\mathrm{C}(6)$ & $-1234(4)$ & $1373(5)$ & $5463(2)$ & $62(1)$ \\
\hline$C(7)$ & $-1280(3)$ & $591(4)$ & $4825(2)$ & $48(1)$ \\
\hline$C(8)$ & $-2028(3)$ & $-659(4)$ & $4583(2)$ & $56(1)$ \\
\hline$C(9)$ & $-1986(4)$ & $-1323(4)$ & $3968(3)$ & $60(1)$ \\
\hline$C(10)$ & $-1194(3)$ & $-743(3)$ & $3574(2)$ & $45(1)$ \\
\hline $\mathrm{C}(11)$ & $.497(3)$ & $1094(3)$ & $4401(2)$ & $35(1)$ \\
\hline$C(12)$ & $345(3)$ & $2343(3)$ & $4635(2)$ & $35(1)$ \\
\hline$C(13)$ & $3505(3)$ & $1237(3)$ & $659(2)$ & $32(1)$ \\
\hline$C(14)$ & $4372(3)$ & $740(3)$ & $3992(2)$ & $42(1)$ \\
\hline $\mathrm{C}(15)$ & $5738(3)$ & $1207(4)$ & $4081(2)$ & $49(1)$ \\
\hline$C(16)$ & $6313(3)$ & $2207(4)$ & $3855(2)$ & $49(1)$ \\
\hline $\mathrm{C}(17)$ & $5505(3)$ & $2736(3)$ & $3536(2)$ & $42(1)$ \\
\hline$C(18)$ & $4102(3)$ & $2279(3)$ & $3432(2)$ & $33(1)$ \\
\hline$C(19)$ & $3364(3)$ & $2842(3)$ & $3037(2)$ & $33(1)$ \\
\hline $\mathrm{C}(20)$ & $1610(3)$ & $3295(3)$ & $2471(2)$ & $40(1)$ \\
\hline$C(21)$ & $720(3)$ & $4042(3)$ & $2761(2)$ & $41(1)$ \\
\hline $\mathrm{Cu}(2)$ & $2554(1)$ & $-1829(1)$ & $1863(1)$ & $36(1)$ \\
\hline $\mathrm{S}(2)$ & $4258(1)$ & $-3794(1)$ & $1952(1)$ & $45(1)$ \\
\hline$O(2 W)$ & $2008(2)$ & $-1705(2)$ & $2802(1)$ & $42(1)$ \\
\hline $0(5)$ & $2076(2)$ & $-296(2)$ & $1790(1)$ & $37(1)$ \\
\hline$O(6)$ & $3023(2)$ & $-3440(2)$ & $1951(1)$ & $49(1)$ \\
\hline$O(7)$ & $4306(4)$ & $-4584(3)$ & $1331(2)$ & $72(1)$ \\
\hline$O(8)$ & $4486(3)$ & $-4292(3)$ & $2511(2)$ & $60(1)$ \\
\hline $\mathrm{O}(9)$ & $-2206(3)$ & $-34(4)$ & $1595(2)$ & $86(1)$ \\
\hline $0(10)$ & $3261(3)$ & $-3166(4)$ & $3521(2)$ & $83(1)$ \\
\hline $\mathrm{N}(4)$ & $600(3)$ & $-2902(3)$ & $1411(2)$ & $45(1)$ \\
\hline $\mathrm{N}(5)$ & $2714(3)$ & $-2152(3)$ & $866(1)$ & $40(1)$ \\
\hline $\mathrm{N}(6)$ & $4489(3)$ & $.779(2)$ & $2253(1)$ & $33(1)$ \\
\hline$C(22)$ & $-411(4)$ & $-3292(4)$ & $1687(2)$ & $62(1)$ \\
\hline $\mathrm{C}(23)$ & $-1704(4)$ & $-3953(5)$ & $1301(3)$ & $83(2)$ \\
\hline$C(24)$ & $-1896(5)$ & $-4181(5)$ & $630(3)$ & $80(2)$ \\
\hline$C(25)$ & $-847(4)$ & $-3789(4)$ & $329(2)$ & $62(1)$ \\
\hline$C(26)$ & $-943(6)$ & $-3993(5)$ & $-377(3)$ & $79(2)$ \\
\hline $\mathrm{C}(27)$ & $97(6)$ & $-3605(5)$ & $-639(2)$ & $78(2)$ \\
\hline $\mathrm{C}(28)$ & $1391(5)$ & $-2954(4)$ & $-238(2)$ & $59(1)$ \\
\hline $\mathrm{C}(29)$ & $2524(6)$ & $-2539(5)$ & $-484(2)$ & $72(1)$ \\
\hline $\mathrm{C}(30)$ & $3728(5)$ & $-1939(5)$ & $-71(2)$ & $66(1)$ \\
\hline$C(31)$ & $3778(4)$ & $-1764(4)$ & $605(2)$ & $50(1)$ \\
\hline $\mathrm{C}(32)$ & I $525(4)$ & $-2739(3)$ & $449(2)$ & $43(1)$ \\
\hline$C(33)$ & $406(4)$ & $-3145(3)$ & $742(2)$ & $48(1)$ \\
\hline$C(34)$ & $2887(3)$ & $615(3)$ & $1644(2)$ & $34(1)$ \\
\hline$C(35)$ & $2392(4)$ & $1323(4)$ & $1276(2)$ & $46(1)$ \\
\hline $\mathrm{C}(36)$ & $3212(4)$ & $2354(4)$ & $1156(2)$ & $55(1)$ \\
\hline$C(37)$ & $4568(4)$ & $2726(4)$ & $1380(2)$ & $54(1)$ \\
\hline$C(38)$ & $5104(4)$ & $2041(3)$ & $1723(2)$ & $44(1)$ \\
\hline $\mathrm{C}(39)$ & $4300(3)$ & $971(3)$ & $1846(2)$ & $34(1)$ \\
\hline$C(40)$ & $4984(3)$ & $311(3)$ & $2195(2)$ & $35(1)$ \\
\hline $\mathrm{C}(41)$ & $5410(3)$ & $-1300(3)$ & $2589(2)$ & $42(1)$ \\
\hline$C(42)$ & $5598(4)$ & $-2399(3)$ & $2110(2)$ & $48(1)$ \\
\hline $\mathrm{C}(43)$ & $-2373(8)$ & $-477(9)$ & $903(3)$ & $136(3)$ \\
\hline $\mathrm{C}(44)$ & $-1251(9)$ & $-456(12)$ & $649(2)$ & $197(6)$ \\
\hline $\mathrm{C}(45)$ & $4153(6)$ & $-2748(6)$ & $4136(3)$ & $96(2)$ \\
\hline $\mathrm{C}(46)$ & $4914(5)$ & $-3532(5)$ & $4293(3)$ & $87(2)$ \\
\hline$O(3 W)$ & $7256(5)$ & $-4159(4)$ & $2849(3)$ & $111(2)$ \\
\hline
\end{tabular}

a. $U_{\text {eq }}$ is defined as one third of the trace of the orthogonalized $U_{\mathrm{ij}}$ tensor.

\section{Acknowledgments}

We thank Foundation of Key Laboratory of Non-ferrous Metal Materials and New Processing Technology, Ministry for Education.

\section{References}

1. L. Casella and M. Gullotti, J. Am. Chem. Soc., 1981, 103,
Table 3 Hydrogen bond lengths $(\AA)$ and bond angles $\left({ }^{\circ}\right)$ of the title compound

\begin{tabular}{lllll}
\hline $\mathrm{D}-\mathrm{H} \cdots \mathrm{A}$ & $\mathrm{D}-\mathrm{H}$ & $\mathrm{H}-\mathrm{A}$ & $\mathrm{D} \cdots \mathrm{A}$ & $\mathrm{DHA}$ \\
\hline $\mathrm{O}(3 \mathrm{~W})-\mathrm{H}(3 \mathrm{WA}) \cdots \mathrm{O}(3)^{\mathrm{a}}$ & $0.68(7)$ & $2.18(7)$ & $2.857(5)$ & $172(8)$ \\
$\mathrm{O}(3 \mathrm{~W})-\mathrm{H}(3 \mathrm{WB}) \cdots \mathrm{O}(8)$ & $1.16(9)$ & $2.16(9)$ & $2.891(6)$ & $118(6)$ \\
$\mathrm{O}(1 \mathrm{~W})-\mathrm{H}(1 \mathrm{WA}) \cdots \mathrm{O}(5)$ & 0.82 & 2.12 & $2.660(3)$ & 123.5 \\
$O(1 \mathrm{~W})-\mathrm{H}(1 \mathrm{WB}) \cdots \mathrm{O}(9)$ & 0.83 & 2.00 & $2.825(4)$ & 175.8 \\
$\mathrm{O}(2 \mathrm{~W})-\mathrm{H}(2 \mathrm{WA}) \cdots \mathrm{O}(1)$ & 0.82 & 2.48 & $2.815(3)$ & 105.7
\end{tabular}

a. Symmetry code: $x+1, y-1, z$.
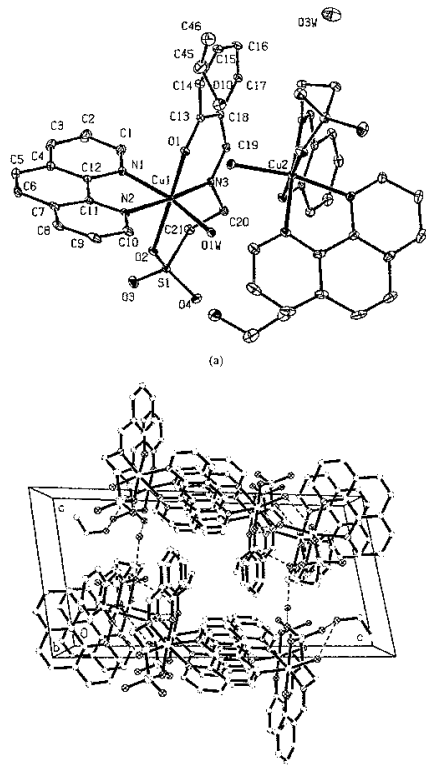

Fig. 2 (a) ORTEP structure of the title compound with atom labeling. The hydrogen atoms are omitted for clarity. (b) Packing diagram of the title compound showing the intermolecular hydrogen bonds and $\pi-\pi$ stacking.

6338.

2. B. Sreenivasulu, M. Vetrichelvan, F. Zhao, S. Gao, and J. J. Vittal, Eur. J. Inorg. Chem., 2005, 4635.

3. M. L. Tong, Y. M. Wu, S. L. Zheng, X. M. Chen, T. Yuen, C. L. Lin, X. Y. Huang, and J. Li, New J. Chem., 2001, 25, 1482.

4. L. S. Hollis, A. R. Amundsen, and E. W. Stern, J. Med. Chem., 1989, 32, 128.

5. G. M. Sheldrick, SHELXL97 and SHELXS, University of Göttingen, Germany, 1997. 